\title{
Assessing the safety and teamwork climate of intensive care staff
}

Sammei Tam RN, RM; MSc Nursing, MSc Health \& Hospital Management, General Manager (Nursing), North District Hospital, Hong Kong

Lorna Kwai Ping Suen RN, RM; BN, MPH, PhD, Associate Professor, The Nethersole School of Nursing, The Chinese University of Hong Kong, Hong Kong

Ka Man Ho RN, RNM; BcN, MHA, Ward Manager, Intensive Care Unit, Prince of Wales Hospital, Hong Kong

Diana Tze Fan Lee RN, RM; RTN, PRD (HCE), MSc, PhD, Chair Professor of Nursing and Director, The Nethersole School of Nursing, The Chinese University of Hong Kong. Assistant Dean, Faculty of Medicine, The Chinese University of Hong Kong

Key Words: intensive care * nurses * safety teamwork

E-mail: lornasuen@cuhk.edu.hk

\section{ESPAÑOL}

Valorando la seguridad y el clima de trabajo en equipo del plantel de cuidado intensivo

\section{Palabras clave}

Cuidado intensivo, enfermeros, seguridad, trabajo en equipo

Resumen

La comunicación es un componente integral de un buen clima de trabajo en equipo.

Los médicos son más positivos en sus percepciones de seguridad y trabajo en equipo en sus unidades que los enfermeros.

- La gestión hospitalaria debería establecer una cultura sin culpa de modo que el plantel pueda discutir abiertamente el asunto cuando el error ocurre.

- Deberían implementarse estrategias para promover el buen trabajo en equipo para mantener la calidad del cuidado y mejorar la seguridad del paciente en unidades de cuidado intensivo.

\section{SUMMARY}

- Communication is an integral component of a good teamwork climate.

- Doctors are more positive in their perceptions of safety and teamwork than nurses in their working units.

- Hospital management should establish a no-blame culture so that staff could openly discuss patient safety issues when errors occur.

- Strategies for promoting good teamwork should be made in order to maintain quality of care and enhance patient safety in intensive care units

\section{INTRODUCTION}

Patient safety is an important component of healthcare quality. Despite the implementation of information technology programs, such as a 2-D barcode identification system, computerised medication order entry, and computerised, web-based incident reporting, many doctors and nurses have expressed deep concerns about making errors (Lau et al. 2005). Being healthcare professionals, we need to assure the public through our dictum, 'First, do no harm'.

Researchers report that a substantial reduction in medical errors is related to human factors, and also noted that teamwork plays an important role in patient safety (Institute of Medicine 2000; Dichter 2003; Kaissi et al. 2003; Thomas et al. 2003). Teamwork enhances effectiveness (Risser et al. 1999), causes fewer and shorter delays, improves morale and job satisfaction, creates lower stress, and improves patient satisfaction (Majzun, 1998; Sexton et al. 2000; Firth-Cozens 2001). Communication is an integral component of a good teamwork climate. It improves efficiency and safety (Boyle \& Kochinda 2004). However, in critical care medicine, communication breakdown is commonly implicated as a source of adverse events. Research indicates that discrepant attitudes about teamwork among critical care nurses and doctors exist. Doctors and nurses in intensive care units (ICU) perceive their teamwork climate differently. Nurses appear to be less satisfied with nurse-physician collaborations than doctors or have lower teamwork climate scores than that of doctors (Thomas et al. 2003; Huang et al. 2007).

A prospective one-year observational study on safety in critical care settings reported that although adverse events and serious errors involving critically ill patients are common and often life-threatening, $45 \%$ of these events are potentially preventable (Rothschild et al. 2005). ICU typically requires physicians to make urgent high-risk decisions that are often based on incomplete data. This may lead to a higher medical error rate compared with other medical settings. Therefore, improving communication during emergency situations by applying the structured teamwork approach could reduce medical errors (Institute of Management 1999; Risser et al. 2000; Sexton et al. 2000).

Effective teamwork can foster patient safety among team members in healthcare delivery (Joint Commission International 2006). A culture of safety enables healthcare personnel to feel they are part 
of a team and to understand how to exchange patient information and other information in a meaningful and respectful way (Colla et al. 2005; Shostek 2007). Therefore, before additional patient safety practices are introduced, the assessment of existing safety and teamwork climate is important.

\section{METHODOLOGY}

\section{Aim of study}

The aim of this study was to measure the perceptions of ICU personnel on safety and teamwork climate in the unit where they were working. Findings of this study would be used in formulating recommendations to improve teamwork and foster patients' safety in an acute care environment.

\section{Setting}

Seven clusters are developed in the Hospital Authority of Hong Kong. Within the targeted cluster, there are seven hospitals and three adult ICUs. The three units vary in unit size and staff-to-patient ratio (with a range from 4.5 to 4.2 nurses per bed according to case complexity), with a total of $48 \mathrm{ICU}$ beds.

All ICUs have daily ward rounds led by senior medical staff, where appropriate decisions are made regarding patient care (for example, investigations, treatment needed). A didactic approach is incorporated into the overall patient management. For the nursing team, each ICU has its own clinical management team led by the ICU Director or Department Operations Manager (head nurse of the unit), and each has relative autonomy to run the unit within the overall framework set by the cluster leadership.

\section{Design and sample}

This was a descriptive survey in which all full-time and qualified nurses and doctors from ICUs within the targeted cluster were recruited in this study. There were 25 ICU doctors and 184 ICU nurses, who constituted the target population within the three ICUs.

\section{Instrument}

The questionnaire used was a modification of the Safety Attitudes Questionnaire and is specific for the ICU setting (SAQ-ICU version) (Sexton et al. 2006). The SAQ-ICU was chosen because of its brevity and wide administration to more than 500 ICUs worldwide (Colla et al. 2005). The original SAQ-ICU includes 64 items and assesses six safety culture factors (teamwork climate, safety climate, stress recognition, job satisfaction, work environment, and perceptions of management). For the purpose of this study, only safety climate factors (items 1-10) and teamwork climate (items 11-20) were used.

The Likert scale for measuring safety and teamwork factors was independently converted to a $0-100$ scale, based on a wellestablished method (Thomas et al, 2003; Huang et al. 2007). Individual items were scored 0 to $100(0=$ strongly disagree; $25=$ disagree; 50 = neutral; 75 = agree; and $100=$ strongly agree). The negatively phrased items were scored in a reverse manner. Thus, high scores reflected positive attitudes and low scores reflected negative attitudes. The maximum mean score for each factor was 100 . In addition, two demographic questions, two items on collaboration and communication with team members (with a Likert scale from very satisfactory to very unsatisfactory), and three dichotomous questions (yes or no) for incident reporting were added to the questionnaire.

\section{Procedures}

Between January 1 and February 1 2008, a self-administered questionnaire with a cover letter was delivered to all doctors and nurses in the three ICUs via their Department Operations Manager. The cover letter stated the purposes of this study and stressed its confidentiality to encourage completion and return. Personal identifiers were not mentioned on the questionnaire, and all completed questionnaires were dropped into a sealed collection box placed at the nursing station in each ICU. Response rates were monitored bi-weekly and communicated to individual ICU Directors and Department Operations Managers to encourage them to increase response in the lowest responding unit.

\section{Data analyses}

Descriptive analysis was performed to determine the frequency of response for each survey item. Mean scores and percent-favourable scores were computed and compared between doctors and nurses. T-test analyses were performed to compare the differences of mean scores between doctors and nurses, whereas chi-square was used to determine the association between percent favourable of safety or teamwork climate and the respondents. All analyses were conducted usin SPSS (Statistical Package for Social Scientists). A p value < 0.05 was set as the level of statistical significance.

Mean score is the average computed by adding each score in the set, then dividing the total by the number of members in the group. It provides a rough point estimate of the factor score of the relevant group. A percent-favourable score is a measure of the percentage of respondents that gave a response to a question that reflected positively, with a mean score higher than or equal to 75 on a 0 to 100 scale, for which 100 was the most favourable (Thomas et al. 2003; Huang et al, 2007). This value was able to reflect more accurately the degree of variability in personnel perceptions within the group.

\section{RESULTS}

A total of 209 questionnaires was distributed to all doctors and nurses in the three ICUs; 135 questionnaires were returned. Among the returned questionnaires, one was excluded due to missing data. An overall response rate of $64.1 \%$ was achieved.

On average, respondents had been working for 8.02 years (ranging from 5.64 to 9.05 years). Doctors had less working experience (5.01 years) than nurses (8.33 years). The mean safety scores and teamwork scores of all respondents were 69.08 and 63.88 , respectively. The percentages of staff members with favourable scores greater than 75 on safety and teamwork factors were less than half, with the percent-favourable for safety factor being 36.07 , whereas it was 15.57 for teamwork factor.

Although all ICU personnel believed the unit was exerting efforts to ensure patient safety (item 2, mean score 77.43 ), the respondents expressed difficulty in discussing errors (item 3, mean score 57.65), nor were they encouraged by colleagues to discuss patient safety issues in their units (item 5 , mean score 63.06). Moreover, many respondents noted it was not easy to share learning points from errors (item 6, mean score 63.43). The respondents were not likely to perceive that interactions in the ICU were collegial (equal level) (item 19, mean score 50.93). The mean score of each item of safety and teamwork climate is displayed in Table 1.

Doctors tended to perceive the factors more positively than nurses. Significant difference in the perception of teamwork between nurses and doctors was noted $(p<0.032)$. In general, ratings for safety climate given by nurses and doctors were higher than for teamwork climate (see Table 2). Overall, the perception of doctor-doctor communication (3.99 out of 5) was slightly higher than nurse-nurse communication (3.77 out of 5). Moreover, doctors indicated more satisfaction with doctor-nurse communication (4.08 out of 5) than nurses' rating of their communication with doctors (3.44 out of 5 ). Of the total questionnaires received, 130 respondents answered the 
question about incident reporting. Only 52 out of 130 indicated they have been involved in any patient safety-related incidents (errors) in the past six months. Regarding incident reporting, only 38 out of 52 respondents $(73 \%)$ reported incidents to their supervisors. Only 46 out of 52 respondents $(88 \%)$ who were involved in at least one safetyrelated incident (error) 'feel comfortable' reporting incidents.

\begin{tabular}{|c|l|c|}
\hline Item & \multicolumn{1}{|c|}{ Safety climate } & $\begin{array}{c}\text { Mean score } \\
\text { (SD) }\end{array}$ \\
\hline 1 & I would feel safe being treated here as a patient & $69.30(15.9)$ \\
\hline 2 & This unit is putting effort to ensure patient safety & $77.43(13.3)$ \\
\hline 3 & In this ICU, it is difficult to discuss errors & $57.65(19.2)$ \\
\hline 4 & $\begin{array}{l}\text { All the staff in my ICU take responsibility for patient } \\
\text { safety }\end{array}$ & $75.17(18.5)$ \\
\hline 5 & $\begin{array}{l}\text { I am encouraged by my colleague to discuss any } \\
\text { patient safety issue }\end{array}$ & $63.06(17.3)$ \\
\hline 6 & $\begin{array}{l}\text { In this ICU, it is easy to share learning points from the } \\
\text { errors }\end{array}$ & $63.43(19.3)$ \\
\hline 7 & $\begin{array}{l}\text { I know the proper channels to direct questions } \\
\text { regarding patient safety in this ICU }\end{array}$ & $67.35(18.5)$ \\
\hline 8 & I know how to report errors that happen in this ICU & $74.44(12.8)$ \\
\hline 9 & $\begin{array}{l}\text { Patient safety is constantly reinforced as a priority in } \\
\text { this ICU }\end{array}$ & $76.12(15.3)$ \\
\hline 10 & $\begin{array}{l}\text { Information obtained through incident reports is used to } \\
\text { make patient care safer in this ICU }\end{array}$ & $69.96(16.5)$ \\
\hline & \multicolumn{1}{c|}{ Teamwork climate } & $63.25(19.07)$ \\
\hline 11 & Nurse input on patient safety recognized in this ICU & $68.28(16.27)$ \\
\hline 12 & Staff input is utilized by this ICU for decision-making & $62.13(18.33)$ \\
\hline 13 & This ICU encourages teamwork among its staff & $67.91(19.54)$ \\
\hline 14 & $\begin{array}{l}\text { In this ICU, I find it difficult to speak up if I perceive a } \\
\text { problem with patient care }\end{array}$ & $60.07(19.66)$ \\
\hline 15 & $\begin{array}{l}\text { Disagreements in this ICU are resolved based on what } \\
\text { is the best for the patient }\end{array}$ & $59.33(16.98)$ \\
\hline 16 & $\begin{array}{l}\text { I have the support I need from other staff to care for } \\
\text { patients }\end{array}$ & $66.42(18.42)$ \\
\hline 17 & $\begin{array}{l}\text { It is easy for staff in this ICU to ask questions when } \\
\text { there is something that they do not understand }\end{array}$ & $68.65(17.53)$ \\
\hline 18 & $\begin{array}{l}\text { The doctors and nurses here work together as a well- } \\
\text { coordinated team }\end{array}$ & $63.03(16.84)$ \\
\hline 19 & $\begin{array}{l}\text { Interactions in the ICU are collegial (equal level), rather } \\
\text { than hierarchical (ranking) }\end{array}$ & $50.93(25.91)$ \\
\hline 20 & $\begin{array}{l}\text { Important issues are communicated well at shift } \\
\text { changes }\end{array}$ & 69.02 \\
\hline
\end{tabular}

Table 1. Mean scores of individual items of safety and teamwork climate

\begin{tabular}{|c|c|c|c|c|}
\hline & \multicolumn{2}{|c|}{ Mean score } & \multicolumn{2}{c|}{ Perecent-favourable (\%) } \\
\hline & Safety & Teamwork & Safety & Teamwork \\
\hline All Doctors & 72.31 & 70.39 & 46.15 & 30.77 \\
\hline All Nurses & 69.05 & 62.87 & 34.71 & 13.43 \\
\hline P value a,b $^{\mathbf{a}, \mathbf{b}}$ & 0.276 & $0.032^{\star}$ & 0.588 & 0.496 \\
\hline
\end{tabular}

Table 2. Mean and percent-favourable scores for safety and

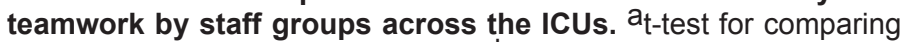
nurses and doctors by mean scores, ${ }^{b}$ Chi-square test for comparing nurses and doctors by percent-favourable, *statistically significant

\section{DISCUSSION}

Doctors rated higher than nurses on safety and teamwork factors. This finding is consistent with overseas studies (Sexton et al. 2000; Kaissi et al. 2003; Thomas et al. 2003; Huang et al. 2007), whereby nurses perceived lower levels of quality of safety and teamwork than doctors. Doctors tended to perceive those factors more positively, most notably for teamwork. The significantly higher teamwork mean scores for doctors $(70.39, p<0.032)$ than for nurses $(62.87)$ indicate that doctors are more satisfied with the team functioning in their working units, and treasure the value of working with others. The smaller numbers of people in the doctor team, as compared with the nurse team, requires them to share responsibilities in caring for patients, makes them know each other better, and contributed to a higher teamwork mean score. On the other hand, ICU nurses often take care of one patient and are more isolated from the rest of the team in their shift. The findings that doctors are more satisfied with doctor-nurse communication than nurse-doctor communication are also in accordance with the findings reported by Thomas et al. (2003) that nurses' rating of the quality of collaboration and communication with physicians (33\% positive ratings) was lower than the latter's ratings of the quality of collaboration and communication with the former's (73\% positive ratings).

The higher mean safety score (72.31) and higher percent-favourable (46.15) for doctors than for nurses $(69.05 ; 34.71)$ indicate that most doctors viewed safety climate more positively than nurses. However, it is important that all staff in ICUs uphold patient safety by reducing errors.

Regarding incident reporting, only $73 \%$ of the respondents reported incidents to their supervisors. Some respondents even expressed that it is difficult to discuss errors or to share learning points from errors. This partly reveals the lack of a blame-free environment, where individuals are able to report errors or near misses without fear of warning or punishment across the units. Additionally, staff might have believed that reporting would not result in any change. Lack of an expectation of collaboration across the team to seek solutions to vulnerabilities or lack of assertiveness to raise patient safety issues lead to negative consequences (Donchin et al. 1995). This is an important finding for management with regard them as role models and encouraging staff to speak up if they notice something abnormal or anything that will affect patient safety. Safety can only be improved when leaders are visibly committed to enable staff to share safety information openly. There should be an understanding among staff that to err is human, and when humans function in complex environments such as ICU, errors will occur (linstitute of Medicine 1999). Senior leaders must drive culture change by demonstrating their own commitment to safety. Otherwise, staff will be unwilling to report adverse events and unsafe conditions.

Sometimes, when incidents occur, the staff involved is often protected and the case will not be disclosed. Most senior team members in the ICU are not open to input from junior members. Over half of ICU staff reported that they find it difficult to discuss errors (Sexton et al. 2000). Such perceptions may reflect insufficient involvement of staff in decision making despite top management's commitment to teamwork. Only when the investigation has been completed and a written report has been submitted to the hospital are learning points shared among staff. Feedback is important for the team to self-correct and learn from experience (Salas et al. 2010). The ICU setting is fast-paced, complex, and commonly requires urgent highrisk decision making. Therefore, it is prudent to develop strategies for staff engagement and involvement in decision making. This will possibly result in higher levels of teamwork and maintenance of the quality of care and patient safety in ICUs. Many organisations have implemented safety briefings which are summaries of incidents that integrate safety issues into daily work handover among ICU staff to help organisations create a culture of safety, reduce risk of errors, and improve the quality of care (Institute for Healthcare Improvement 2008).

The ICU staff's manner of rating of the interactions in ICUs is rather hierarchical. Relative to doctors, nurses were more reluctant to speak up. Perhaps nurses' inputs are not that well accepted (Thomas et al. 
2003). This might be attributed to fundamental differences between nurses and doctors, such as status, authority, gender, training, and patient-care responsibilities (Makary et al. 2006). Historically, nurses can be hesitant to confront ICU doctors on issues of care treatment because they might have less training or experience in dealing with a patient's medical condition. However, in reality, nurses are well positioned to detect abnormal effects and test system failures. If they are not willing to speak up, this might have harmful consequences for patients, as well as negative impact for ICU personnel. All of these may result in personnel's perception of hierarchical interactions in ICU and contribute to dissatisfaction with their profession. Developing initiatives to promote a positive working environment, in which good teamwork enhances job satisfaction and improves nurse morale, is a new challenge to all ICU managers.

Communication is an integral component of a culture of good teamwork that improves efficiency and safety (Boyle \& Kochinda 2004). To improve teamwork, personnel should be complemented with appropriate knowledge and skills in communication by additional training (Schull et al. 2001). Efforts should be better directed to personnel training, focusing on communication and collaboration among staff.

\section{FURTHER RESEARCH}

The primary limitation of this study was the small sample size. Generalisation is also limited by our single hospital cluster study design. Further studies on a larger scale can explore the variations of personnel perception of safety and teamwork climate among different hospitals.

The effect of training interventions and the relationship between culture and outcomes (for example, reduction of patient length of stay, adverse events, and staff turnover) can also be explored. With reference to other studies, multidimensional assessment includes perceptions of management, stress recognition, job satisfaction, and work environment (Kaissi et al. 2003; Huang et al. 2007). Although multidimensional assessment is more labour-intensive, it provides specific recommendations for unit-based cultural interventions.

\section{CONCLUSIONS}

This is the first study to examine staff perceptions of teamwork and safety in ICUs; hence, findings from the current study provide management and leadership with a starting point for understanding patient safety culture issues in the future planning of improvement programs.

In general, doctors were more positive in their perceptions of safety and teamwork than nurses in their working units. Overall, ICU personnel considered that communication was satisfactory; however, doctors indicated more satisfaction with doctor-nurse communication than nurses' rating of nurse-doctor communication.

ICU personnel should be complemented with appropriate knowledge and skills in communication by additional training to improve teamwork. Moreover, team-based training in ICU is necessary because it is likely to fill gaps in the perceptions between doctors and nurses. Findings regarding some respondents who did not report incidents that occurred indicate that more efforts should be directed to create environments that encourage staff to speak up and easily share learning points from errors. Hospital management should make deliberate decisions about disciplinary actions consistent with a no-blame culture so that staff could openly discuss patient safety issues when errors occur. Safety briefings can be carried out to increase staff awareness of patient safety. In addition, safety walkarounds can be put into practice to discuss safety-related issues with frontline staff. All these actions will certainly help promote a positive working environment, result in good teamwork, and maintain quality of care and patient safety in ICUs.

\section{REFERENCES}

Boyle D, Kochinda C (2004). Enhancing collaborative communication of nurse and physician leadership in two intensive care units. Journal of Nursing Administration 34 (2), 60-70.

Colla J, Bracken A, Kinney L, Weeks W (2005). Measuring patient safety climate: a review of surveys. Quality and Safety in Health Care 14 (5), 364-366.

Dichter JR (2003). Teamwork and hospital medicine. Critical Care Nurse 23 (3), 8, 10-11.

Donchin Y, Gopher D, Olin M, Badihi Y, Sprung C, Pizov R, Cotev A (1995). A look into the nature and causes of human errors in the intensive care units. Critical Care Medicine 23 (2), 294-300.

Firth-Cozens J (2001). Cultures for improving patient safety through learning: the role of teamwork. Quality in Health Care 10 (Suppl 2), ii26-31.

Huang D, Clermont G, Sexton J, Karlo C, Miller R, Weissfeld L, Rowan K, Angus D (2007). Perceptions of safety culture vary across the intensive care units of a single institution. Critical Care Medicine 35 (1), 165-176.

Institute for Healthcare Improvement (2008). Develop a Culture of Safety [online]. Available at: http://www.ihi. org/lHI/Topics/PatientSafety/SafetyGeneral/Changes/ Develop+a+Culture+of+Safety.htm [Accessed December 2010].

Institute of Medicine (1999). Institute of Medicine Report [online]. Available at: http://md-jd.info/abstract/Institute-of-MedicineReport.html. [Accessed December 2010].

Institute of Medicine (2000). To Err Is Human: Building a Safer Health System [online]. Available at: http://books.nap.edu/ openbook.php?record_id=9728\&page=17. [Accessed December 2010]

Joint Commission International (2006). Innovative Solutions to Medical Liability [online]. Available at: http://www.jointcommission. org/NewsRoom/OnCapitolHill/mvotestimony+_071306.htm. [Accessed November 2007].

Kaissi A, Johnson T, Kirschbaum M. (2003). Measuring teamwork and patient safety attitudes of high-risk Areas. Nursing Economics 21 (5), 211-218.

Lau L, Chiu S, Wong M (2005). To break the wall of silence - a long journey to strive on. In: Proceedings of the Hospital Authority Convention 2005, Hong Kong, p 63. [Online]. Available at: http:// www.ha.org.hk/haconvention/hac2005proceedings/doc/npe6-1. pdf.

Majzun $R$ (1998). The role of teamwork in improving patient satisfaction. Group Practice Journal 47 (4), 12-16.

Makary M, Sexton J, Freischlag J, Holzmueller C, Millmann E, Rowen L, Pronovost P (2006). Operating room teamwork among physicians and nurses: teamwork in the eye of the beholder. Journal of American College of Surgeons 202 (5), 746-752.

Risser D, Simon R, Rice M, Salisbury M (2000). A Structured Teamwork System to Reduce Clinical Errors. In: L. Spath (Ed.), Error Reduction in Health Care: A Systems Approach to Improving Patient Safety (pp. 235-276). New York: Jossey-Bass.

Risser T, Rice M, Salisbury M, Simon R, Jay GD, Berns SD (1999). The potential for improved teamwork to reduce medical errors in the emergency department. Annals of Emergency Medicine 34 (3), 373-383. 
Rothschild J, Landrigan C, Cronin J, Kaushal R, Lockley S, Burdick E, Stone P, Lilly C, Katz J, Czeisler C, Bates D (2005). The Critical Care Safety Study: The incidence and nature of adverse events and serious medical errors in intensive care. Critical care Medicine 33 (8), 1694-1700.

Sales E, Rosen MA, King H (2010). Managing teams managing crises: principles of teamwork to improve patient safety in the Emergency Room and beyond. Theoretical Issues in Ergonomics Science 8 (5), 381-394.

Schull M, Ferris L, Tu J, Hux J, Redelmeier D (2001). Problems for clinical judgement: thinking clearly in an emergency. Canadian Medical Association Journal 164 (8), 1170-1175.
Sexton J, Helmreich R, Neilands T, Rowan K, Vella K, Boyden J, Roberts P, Thomas E (2006). The safety attitudes questionnaire: psychometric properties, benchmarking data, and emerging research. BMC Health Services Research 03 April, 6 (44).

Sexton J, Thomas E, Helmreich R (2000). Error, stress, and teamwork in medicine and aviation: cross sectional surveys. BMJ 320 (7237), 745-749.

Shostek K (2007). Critical Care Safety Essentials. Patient Safety and Quality Healthcare 4 (5), 44-48.

Thomas E, Sexton J, Helmreich R (2003). Discrepant attitudes about teamwork among critical car nurses and physicians. Critical Care Medicine 31 (3), 956-959. 